\title{
Paradigma Deese-Roediger-McDermott: Efeito da Evocação Prévia e Tipo de Tarefa de Memória
}

\author{
Eduarda Pimentel ${ }^{1}$ \\ Faculdade de Filosofia de Braga, Universidade Católica Portuguesa \\ Pedro B. Albuquerque \\ Escola de Psicologia, Universidade do Minho
}

\begin{abstract}
RESUMO - Pretendeu-se averiguar se a activação dos itens críticos no paradigma de Deese-Roediger-McDermott também ocorreria numa tarefa de completamento. Para analisar a contaminação explícita explorámos a existência de resultados dissociados em função da manipulação do nível de processamento. Na tarefa de completamento, a primação semântica foi estatisticamente superior à primação directa. A ausência do efeito do nível de processamento demonstra que o teste foi de memória implícita. Também avaliámos o impacto de uma tarefa de evocação numa tarefa de memória posterior. Verificámos que a evocação prévia anulou o efeito do nível de processamento na tarefa de reconhecimento. Na tarefa de completamento de inícios de palavras, o incremento de inícios de palavras completados com associados só foi expressivo quando as palavras foram codificadas superficialmente.
\end{abstract}

Palavras-chave: falsas memórias; paradigma DRM; teste de memória; nível de processamento; recordação prévia

\section{Deese-Roediger-McDermott Paradigm: Effect of Previous Recall and Type of Memory Task}

\begin{abstract}
This study aimed to verify whether the activation of critical items in the Deese-Roediger-McDermott paradigm of producing false memories could also occur in the word stem completion task. The finding that the level of processing did not seem to have any effect on the word stem completion task supported the conclusion that the stem completion task was in fact an implicit memory test. The impact of a previous recall in a following memory task was also evaluated. The results indicated that the previous recall inhibited the effect of the processing level in the recognition task. In the word stem completion task the increase of stems completed with associates was only relevant when words were encoded superficially.
\end{abstract}

Keywords: false memories; DRM paradigm; memory test; level of processing; previous recall

As falsas memórias, também denominadas distorções ou ilusões de memória, correspondem à recordação, parcial ou totalmente alterada, de acontecimentos passados (Roediger \& McDermott, 2000). Este efeito tem sido amplamente estudado a partir de um procedimento experimental de associados convergentes concebido por Deese (1959) e posteriormente desenvolvido por Roediger e McDermott (1995). O paradigma de associados convergentes, ou paradigma DRM (Deese/ Roediger/McDermott) como é mais conhecido, consiste na apresentação de listas de palavras, cada uma daquelas associadas a um item não apresentado - item crítico - que constitui o tema central da lista (e.g., "bolo", "açúcar", "chocolate", "algodão", "agradável", etc., associados ao item crítico "doce") seguidas, em geral, de uma tarefa de evocação ("recall"), por lista, e/ou de uma tarefa de reconhecimento (e.g., Albuquerque \& Pimentel, 2005; Pinho, Simões, Beato, \& Díez, 2004; Stein, Feix, \& Rohenkohl, 2006; Stein \& Pergher, 2001). Não obstante os participantes serem instruídos a não tentarem adivinhar as palavras apresentadas, observa-se um efeito robusto de formação de ilusões de memória nas tarefas de evocação e de reconhecimento. Como exemplo, no estudo de Roediger e McDermott (1995, Experimento 1)

1 Endereço para Correspondência: Faculdade de Filosofia, Universidade Católica Portuguesa, Praça da Faculdade, nº 1. Braga. Portugal. 4710297 E-mail: epimentel@braga.ucp.pt aqueles autores obtiveram uma média de intrusão de itens críticos e de falsos alarmes relativos a itens críticos de $40 \%$ e $84 \%$, respectivamente.

Genericamente, os estudos realizados com o paradigma DRM têm procurado responder a três questões centrais: se o efeito de falsas memórias se deve a processos mnésicos - que poderão ocorrer durante a codificação, recuperação ou em ambas as fases -, ou a processos decisórios, isto é, à tentativa dos participantes em adivinhar ou inferir estrategicamente as palavras estudadas apenas com base na sua relação com a lista apresentada; se a activação dos itens críticos poderá resultar de processos não conscientes durante o estudo das listas; e finalmente, se os itens críticos são também recuperados em tarefas de memória implícita. $\mathrm{O}$ estudo que aqui apresentamos procurou contribuir para dar resposta a esta última questão.

Designam-se de tarefas de memória implícita as medidas de memória que se caracterizam por não fazerem apelo à recordação intencional ou consciente do episódio em que o material foi processado (Schacter, 1987). Este tipo de medidas não se diferencia pela actividade realizada, mas antes pelas instruções fornecidas para a sua concretização (Albuquerque, 2001). Por conseguinte, a mesma tarefa de memória pode ser realizada de forma explícita ou implícita. Por exemplo, o 
completamento de inícios de palavras² (e.g., ALM__ início de "almoço") pode consistir numa tarefa explícita quando os participantes são orientados a completarem os inícios de palavras com base em palavras previamente apresentadas; ou implícita quando é solicitado o completamento com a primeira palavra que ocorre à mente. Note-se que, ao contrário do que sucede na primeira situação, na segunda, a instrução omite a ligação existente entre a realização da tarefa proposta e o episódio prévio de codificação. Sendo assim, este tipo de tarefas de memória podem consistir em actividades tão diversas como: a identificação de um estímulo apresentado rapidamente; o completamento de inícios ou de fragmentos de palavras com a primeira palavra que ocorre à mente; a decisão ou juízo acerca das propriedades de palavras ou de objectos; ou a produção de itens de uma dada categoria como resposta a uma pista (Schacter, Dobbins, \& Schnyer, 2004).

Nestas tarefas, em que não é pedido explicitamente aos participantes a recordação do material previamente apresentado mas, tão somente, o desempenho de uma tarefa aparentemente não relacionada com o episódio de processamento, é constatável uma melhoria na capacidade de identificar, produzir ou classificar um item como resultado de um encontro prévio com esse mesmo item ou outro relacionado (Schacter, Gallo, \& Kesinger, 2007). Esse efeito de facilitação é conhecido na literatura como efeito de primação ("priming"). A título de exemplo, em tarefas de completamento de inícios de palavras e de completamento de fragmentos a primação traduz-se não só num menor tempo de completamento com as palavras vistas anteriormente, do que com outras palavras que não foram apresentadas, como em mais completamentos das pistas com as palavras vistas anteriormente.

No contexto do paradigma DRM é possível constatar duas modalidades de primação: primação por repetição ou primação directa ("direct priming"), e primação indirecta ("indirect priming"). Esta distinção baseia-se na relação entre o estímulo que gera o efeito de facilitação - primo ("prime") - e a resposta subsequente - alvo - . Enquanto que a primação directa se verifica quando a melhoria no processamento de um estímulo decorre do encontro recente com esse mesmo estímulo (Schacter, 1987), isto é, sempre que o primo e o alvo são os mesmos (e.g., primo - "cadeira", alvo - "cadeira"), a primação indirecta tem lugar quando o item estudado e o alvo são de algum modo relacionados, quer pela aparência, pelo som ou pelo seu significado (Roediger, 1990; Roediger \& McDermott, 1993) (e.g., primo - “cadeira", alvo - "mesa"). Deste modo, no paradigma DRM a primação directa reporta-se à primação dos associados (i.e., os itens previamente apresentados) enquanto que a primação indirecta se refere à primação dos itens críticos (i.e., os itens relativamente aos quais os estímulos apresentados estão associados). Este último tipo de primação, no qual o primo e o alvo estão semanticamente relacionados, denomina-se de primação semântica ("semantic priming").

2 Traduzimos a expressão "stem completion task" por tarefa de completamento de inícios de palavras e não de radicais de palavras pois, muito embora, a tradução de "stem" seja "radical" (de palavra), nem sempre as primeiras três letras de uma palavra correspondem ao seu radical (i.e., parte da palavra que permanece igual quando esta é alterada para diferentes terminações) (e.g., o radical de "amor" é AM e não AMO).
Em tarefas memória implícita, os primos podem ser apresentados acima ou abaixo do limiar de consciência (pré-conscientemente). Na primação subliminar, a duração do processamento subliminar difere em função do tipo de estímulo, sendo que no caso de palavras (os estímulos usados no paradigma DRM) a exposição varia entre 25 a 50 milissegundos (Busnello, Stein, \& Salles, 2008 conforme citado em Busnello, 2010; Foster, 1999 conforme citado em Busnello, 2010).

O paradigma DRM tem sido adaptado para a utilização de tarefas perceptivas de memória implícita, também designadas de tarefas dirigidas pelos dados, pelo o facto de serem fortemente influenciadas pelas semelhanças físicas dos estímulos das fases de estudo e de teste (Roediger \& Blaxton, 1987; Roediger \& McDermott, 1993). Mais especificamente, no que se refere às tarefas de completamento de inícios de palavras os estudos têm demonstrado, na sua maioria, a primação significativa do item crítico (e.g. Hicks \& Starns, 2005, Experimento 1; McKone \& Murphy, 2000, Experimentos 1 e 3; Smith, Gerkens, Pierce, \& Choi, 2002, Experimentos 2 e 3; Tajika, Neumann, Hamajima, \& Iwahara, 2005) havendo, contudo, outros estudos que apontam um efeito de primação semântica apenas marginalmente significativo, ou mesmo não significativo (e.g. McBride, Coane, \& Raulerson, 2006, Experimentos 1 e 3; McDermott, 1997, Experimento 3).

Notamos que a activação significativa do item crítico - tema central de cada lista - é um dado inconsistente se atendermos ao facto das tarefas de completamento de inícios de palavras serem consideradas perceptivas por apelarem à ortografia das palavras e não ao seu significado (e.g. Roediger \& McDermott, 1993; Roediger, Weldon, \& Challis, 1989). Por conseguinte, e dado que o item crítico não é apresentado aos participantes, carece de uma representação perceptiva, não devendo, como tal, ocorrer o efeito de primação directa. Por conseguinte, se a activação semântica do item crítico neste tipo de tarefas de memória implícita, por si só, não produz efeito de primação perceptiva (McDermott, 1997), seria de esperar somente a primação dos associados. Mais, atendendo a que a activação semântica é um fenómeno de curta duração, não deveria manifestar-se no paradigma DRM uma vez que neste procedimento o estudo das listas e o teste é mediado por diversos itens e intervalos de retenção relativamente longos.

Sendo assim, e dado que vários estudos mostram a activação significativa do item crítico nesse tipo de tarefas é possível equacionar diferentes explicações: a contaminação explícita do teste; a activação do item crítico durante a codificação dos associados (como sugere a hipótese da activação implícita) sendo essa presença imaginada responsável pelo efeito de primação por repetição do item crítico (para além da primação semântica); as tarefas em causa não serem puramente perceptivas, o que é suportado por alguns estudos em que não foi obtido o efeito de primação semântica do item crítico em tarefas que, comparativamente às de completamento de inícios de palavras, privilegiarão o acesso às características físicas dos estímulos codificados (i.e. tarefas de decisão lexical e de identificação perceptiva) (e.g., McKone, 2004; Zeelenberg \& Pecher, 2002).

Com o presente estudo pretendemos testar se os itens críticos seriam recuperados numa tarefa de memória implí- 
cita de completamento de inícios de palavras, sem recurso a estratégias de recordação explícita.

Os estudos com tarefas de memória implícita no paradigma DRM têm recorrido a diferentes metodologias para prevenir a contaminação com estratégias de recuperação explícitas, designadamente a adopção de uma modalidade de resposta rápida. Outras metodologias são usadas a posteriori como forma de averiguar se houve, ou não, contaminação explícita. Neste âmbito, tem sido privilegiado o preenchimento de um questionário pós-experimental sobre as estratégias de memória empregues pelos participantes durante o completamento dos inícios de palavras (e.g., McKone \& Murphy, 2000, Experimento 1; Tajika et al., 2005; Hicks \& Starns, 2005, Experimento 1), metodologia que consideramos não ser suficientemente eficaz na identificação de todos os casos de recuperação explícita, pois os participantes poderão negar a utilização desta estratégia evitando, assim, assumir implicitamente o incumprimento das instruções do teste.

Um método alternativo, usado por Hicks e Starns (2005, Experimento 1) e por McBride et al. (2006, Experimento 1), consiste na manipulação do nível de processamento. Dado que esta variável afecta apenas a memória explícita - deixando inalterada a memória implícita - pode ser usada para analisar se a tarefa em causa foi realizada de modo explícito ou implícito. Os referidos estudos manipularam o nível de processamento, mas somente o de McBride et al. (2006) comparou o seu efeito numa tarefa de memória explícita. Naqueles estudos verificou-se a ausência do efeito do nível de processamento na melhoria da recuperação implícita de associados e de itens críticos confirmando, assim, que a recuperação foi não consciente efeito que, como seria de esperar, foi obtido na condição de recuperação explícita no estudo de McBride et al. (2006). Porém, somente na experiência de Hicks e Starns (2005) se observou o efeito de primação do item crítico, além disso, no estudo de McBride et al. (2006) as autoras puderam verificar, com base nas respostas ao questionário de estratégias de memória realizado após o teste, que a primação do item crítico foi somente significativa no grupo classificado como "consciente do teste" (i.e., constituído por participantes que se aperceberam, no decurso do mesmo, que alguns inícios de palavras correspondiam às três primeiras letras das palavras anteriormente vistas, pese embora, não tivessem tentado usar nas suas respostas essas palavras).

Tal como nos estudos de Hicks e Starns (2005, Experimento 1) e de McBride et al. (2006, Experimento 1), também nós analisámos a eventual contaminação explícita do teste recorrendo à manipulação do nível de processamento das palavras apresentadas.

Finalmente, um segundo objectivo do presente estudo reportou-se à análise do efeito de activação por evocação prévia na produção de memórias verdadeiras e de falsas memórias numa tarefa de memória explícita (reconhecimento) e noutra de memória implícita (completamento de inícios de palavras). Notamos que os estudos com o paradigma DRM têm analisado exclusivamente o efeito daquela variável no desempenho duma tarefa de memória explícita posterior - reconhecimento (e.g., Roediger \& McDermott, 1995, Experimento 2; Stein \& Pergher, 2001).

Em suma, o presente estudo procurou testar várias hipóteses. Assim, e relativamente ao efeito do nível de processamento pressupôs-se que o processamento profundo (comparativamente ao superficial) favoreceria a recuperação de associados nas tarefas de evocação e de reconhecimento; porém, não produziria uma melhoria na activação de associados na tarefa de completamento de inícios de palavras. No que se refere ao impacto da evocação prévia supusemos que esta favoreceria a recuperação de associados e de itens críticos na tarefa de reconhecimento; bem como, a activação do mesmo tipo de itens na tarefa de memória implícita de completamento de inícios de palavras. Finalmente, relativamente ao efeito de falsas memórias produzido pelas listas de associados convergentes (listas DRM) esperávamos que se verificasse a activação quer de associados, quer de itens críticos na tarefa de inícios de palavras.

\section{Método}

\section{Participantes}

A experiência contou com a colaboração de 80 estudantes, dos quais 62 frequentavam a Universidade do Minho e 18 a Universidade Católica Portuguesa (Centro Regional de Braga). Os participantes, cujas idades variaram entre os 18 e os 33 anos, tinham em média 20,8 anos sendo o desvio padrão de 3,17 anos. Relativamente ao género, $83,8 \%$ dos estudantes eram de sexo feminino e $16,2 \%$ de sexo masculino.

A colaboração no estudo foi voluntária, tendo sido os alunos que frequentavam a Universidade do Minho recompensados com créditos a uma unidade curricular.

\section{Delineamento}

O estudo envolveu o seguinte plano factorial misto: 2 (nível de processamento: superficial vs. profundo) x 2 (evocação prévia: presente vs. ausente) x 2 (tipo de tarefa de memória: implícita vs. explícita) com medidas repetidas no primeiro factor.

A existência de duas variáveis inter-participante com duas condições deu origem à constituição de quatro grupos experimentais: (1) recuperação explícita com evocação prévia; (2) recuperação implícita com evocação prévia; (3) recuperação explícita sem evocação prévia; (4) recuperação implícita sem evocação prévia.

Os primeiros dois grupos efectuaram a evocação dos associados após a apresentação de cada lista, distinguindo-se pelo facto do primeiro ter posteriormente realizado uma tarefa de reconhecimento (explícita) e o segundo uma tarefa de completamento de inícios de palavras (implícita). Os últimos dois grupos realizaram uma tarefa distractiva de cálculo mental após a apresentação de cada lista, sendo que posteriormente um deles foi sujeito a uma tarefa de reconhecimento e o outro a uma tarefa de completamento de inícios de palavras.

As variáveis dependentes foram três e consistiram no grau de retenção obtido nas tarefas de evocação e de reconhecimento e no completamento de inícios de palavras. $\mathrm{Na}$ tarefa de evocação foi medida a quantidade de palavras correctamente evocadas (associados), itens críticos (palavras 
não apresentadas que correspondiam aos temas das listas) e de intrusões (outras palavras não apresentadas evocadas que não eram os itens críticos). Na tarefa de reconhecimento calculou-se a quantidade de êxitos (palavras apresentadas e reconhecidas como tal), falsos alarmes relativos a itens críticos, falsos alarmes relativos a palavras associadas não apresentadas e falsos alarmes relativos a palavras não associadas não apresentadas. Finalmente, na tarefa de completamento de inícios de palavras determinou-se a percentagem de completamentos com associados e itens críticos.

\section{Materiais e equipamento}

A codificação dos estímulos, a tarefa distractiva intercalar, assim como, as tarefas de memória de reconhecimento e de completamento de inícios de palavras foram realizadas com recurso a um computador portátil e programadas na aplicação informática SuperLab Pro 2.0.4 (Cedrus Corporation, San Pedro, CA, USA).

Foram apresentadas aos quatro grupos experimentais as mesmas oito listas de 12 palavras, bem como, uma lista de treino, retirada do artigo de Albuquerque (2005). A composição das listas e dos respectivos itens críticos encontra-se na Tabela 1 .

Como é possível observar, os itens apresentados de uma lista não se repetem nas outras. Relativamente às listas originais foi necessário substituir, ainda que pontualmente, alguns associados por outros da mesma lista que se situavam abaixo da 12. " posição serial (e.g., na Lista 4 o item "costura" foi substituído pelo associado "objecto", dado que "costura" e "coser", que pertencente à mesma lista, iniciam com as mesmas primeiras três letras - COS__ _ Este procedimento resultou da necessidade dos inícios de palavras (relativos aos associados, itens críticos, palavras associadas não apresentadas e de palavras não associadas não apresentadas) usados na tarefa de completamento deverem corresponder, apenas, a uma palavra. Este constitui um procedimento metodológico fundamental para o cálculo da primação directa e da primação semântica.
A lista de palavras para reconhecimento era composta por 56 itens: 16 palavras apresentadas, duas por lista (uma processada de modo superficial e outra de modo profundo); oito itens críticos; 16 palavras associadas não apresentadas, duas por lista; 16 palavras não associadas não apresentadas. A Tabela 2 contém as palavras que integraram a tarefa de reconhecimento.

A tarefa de completamento de inícios palavras era constituída pelos inícios das mesmas palavras que compunham a tarefa de reconhecimento. Na Tabela 2 indica-se a percentagem de completamento de inícios de palavras relativos a cada uma das palavras (linha de base), reportando-se esses valores exclusivamente ao completamento das palavras em causa e não à de palavras próximas quer do ponto de vista semântico, quer morfológico. Por exemplo, relativamente ao associado não apresentado "engorda" indicamos a percentagem de completamentos de "ENG__ com essa mesma palavra $(0 \%)$ e não com "engordar" $\overline{(4,3} \%)$ (cf. Pimentel \& Albuquerque, 2007).

\section{Procedimento}

Todos os participantes foram instruídos de que seriam apresentadas palavras no ecrã de um computador e que após a apresentação de cada uma delas surgiria uma questão. Algumas vezes ser-lhes-ia perguntado se a palavra vista anteriormente continha um determinado número de vogais; outras vezes, seria visível uma frase com um espaço em branco que corresponderia a uma palavra que faltava, sendo a tarefa dos participantes dizer se a palavra vista previamente completava correctamente a frase apresentada. Para responder a ambos os tipos de questões os participantes deveriam utilizar as teclas "S" (de "sim") e "N" (de "não"), procurando responder correctamente e o mais rápido possível. Em todas as listas, os participantes codificaram cada palavra apresentada num diferente nível de processamento: superficial ou profundo. Embora não fossem informados, o nível de processamento de cada palavra das listas ia alternando (superficial - profunda - superficial ...). Assim sendo, e uma vez que todas

Tabela 1. Listas de associados e respectivos itens críticos (em itálico)

\begin{tabular}{|c|c|c|c|c|c|c|c|c|}
\hline Lista Treino & Lista 1 & Lista 2 & Lista 3 & Lista 4 & Lista 5 & Lista 6 & Lista 7 & Lista 8 \\
\hline alto & frio & lento & doce & agulha & raiva & caneta & ladrão & sono \\
\hline baixo & inverno & rápido & bolo & picada & ódio & escrever & polícia & cama \\
\hline montanha & neve & caracol & açúcar & linha & fúria & lápis & prisão & prazer \\
\hline torre & casaco & devagar & chocolate & coser & sentimento & azul & banco & descanso \\
\hline inatingível & roupa & tartaruga & algodão & palheiro & medo & tinta & assalto & noite \\
\hline enorme & desconforto & vagaroso & agradável & objecto & cólera & papel & dinheiro & muito \\
\hline pico & cachecol & preguiçoso & saboroso & dedal & vacina & esferográfica & gatuno & almofada \\
\hline céu & arrepio & demorado & rebuçado & alfinete & angústia & preta & fuga & insónia \\
\hline altura & tremer & molengão & guloso & fina & nervos & estudo & cadeia & acordado \\
\hline cimo & agasalho & lesma & sobremesa & bordar & zangado & trabalho & criminoso & desperto \\
\hline avião & chuva & inactivo & gostoso & injecção & violência & letra & homem & relaxar \\
\hline difícil & aquecedor & irritante & morango & afiada & agressão & aulas & casa & repouso \\
\hline elevado & camisola & alentejo & azedo & sangue & doença & folha & arma & manhã \\
\hline
\end{tabular}


Tabela 2. Itens críticos (IC), associados (AS), palavras associadas não apresentadas (Anap) e palavras não associadas não apresentadas (NAnap) usados na tarefa de reconhecimento e os respectivos inícios de palavras (primeiras três letras de cada item) usados na tarefa de completamento; percentagens de completamento com aqueles itens (linha de base)

\begin{tabular}{|c|c|c|c|c|c|c|c|c|c|c|c|c|}
\hline Lista & IC & $\% \mathrm{C}$ & Início & AS & $\% \mathrm{C}$ & Início & Anap & $\% \mathrm{C}$ & Início & NAnap & $\% \mathrm{C}$ & Início \\
\hline 1 & frio & 21,6 & fri & $\begin{array}{l}\text { inverno } \\
\text { roupa }\end{array}$ & $\begin{array}{l}43,1 \\
51,2\end{array}$ & $\begin{array}{l}\text { inv } \\
\text { rou }\end{array}$ & $\begin{array}{l}\text { cobertor } \\
\text { constipação }\end{array}$ & $\begin{array}{l}9,3 \\
0\end{array}$ & $\begin{array}{l}\mathrm{cob} \\
\mathrm{con}\end{array}$ & \multirow{8}{*}{$\begin{array}{l}\text { dança } \\
\text { clássica } \\
\text { ritmo } \\
\text { refeição } \\
\text { adega } \\
\text { cumprimento } \\
\text { namorado } \\
\text { miséria } \\
\text { necessidade } \\
\text { tonturas } \\
\text { brincar } \\
\text { grande } \\
\text { tronco } \\
\text { dura } \\
\text { fedorento } \\
\text { sujidade }\end{array}$} & \multirow{8}{*}{$\begin{array}{l}16,3 \\
9,3 \\
11,8 \\
12,0 \\
17,3 \\
12,0 \\
14,9 \\
10,9 \\
10,0 \\
7,8 \\
19,6 \\
11,6 \\
9,3 \\
7,3 \\
20,9 \\
21,6\end{array}$} & \multirow{8}{*}{$\begin{array}{l}\text { dan } \\
\text { clá } \\
\text { rit } \\
\text { ref } \\
\text { ade } \\
\text { cum } \\
\text { nam } \\
\text { mis } \\
\text { nec } \\
\text { ton } \\
\text { bri } \\
\text { gra } \\
\text { tro } \\
\text { dur } \\
\text { fed } \\
\text { suj }\end{array}$} \\
\hline 2 & lento & 14,6 & len & $\begin{array}{l}\text { caracol } \\
\text { vagaroso }\end{array}$ & $\begin{array}{l}4,2 \\
0\end{array}$ & car & $\begin{array}{l}\text { atrasado } \\
\text { autocarro }\end{array}$ & $\begin{array}{l}4,7 \\
7,8\end{array}$ & atr & & & \\
\hline 3 & doce & 5,9 & doc & $\begin{array}{l}\text { bolo } \\
\text { algodão }\end{array}$ & $\begin{array}{l}21,6 \\
0\end{array}$ & $\begin{array}{l}\text { bol } \\
\text { alg }\end{array}$ & $\begin{array}{l}\text { engorda } \\
\text { mousse }\end{array}$ & $\begin{array}{l}0 \\
8,5\end{array}$ & $\begin{array}{l}\text { eng } \\
\text { mou }\end{array}$ & & & \\
\hline 4 & agulha & 29,4 & agu & $\begin{array}{l}\text { coser } \\
\text { palheiro }\end{array}$ & $\begin{array}{l}13,1 \\
0\end{array}$ & $\begin{array}{l}\mathrm{cos} \\
\mathrm{pal}\end{array}$ & $\begin{array}{l}\text { perigo } \\
\text { tesoura }\end{array}$ & $\begin{array}{l}0 \\
20,0\end{array}$ & $\begin{array}{l}\text { per } \\
\text { tes }\end{array}$ & & & \\
\hline 5 & raiva & 30,2 & rai & $\begin{array}{l}\text { sentimento } \\
\text { medo }\end{array}$ & $\begin{array}{l}14,0 \\
52,9\end{array}$ & sen & $\begin{array}{l}\text { revolta } \\
\text { gritar }\end{array}$ & $\begin{array}{l}12,8 \\
23,4\end{array}$ & $\begin{array}{l}\text { rev } \\
\text { gri }\end{array}$ & & & \\
\hline 6 & caneta & 4,7 & can & $\begin{array}{l}\text { escrever } \\
\text { tinta }\end{array}$ & $\begin{array}{l}2,4 \\
38,0\end{array}$ & $\begin{array}{l}\text { esc } \\
\text { tin }\end{array}$ & $\begin{array}{l}\text { apontamentos } \\
\text { estudante }\end{array}$ & $\begin{array}{l}4,2 \\
4,0\end{array}$ & $\begin{array}{l}\text { apo } \\
\text { est }\end{array}$ & & & \\
\hline 7 & ladrão & 21,7 & lad & $\begin{array}{l}\text { polícia } \\
\text { assalto }\end{array}$ & $\begin{array}{l}17,4 \\
0\end{array}$ & $\begin{array}{l}\text { pol } \\
\text { ass }\end{array}$ & $\begin{array}{l}\text { punir } \\
\text { delinquência }\end{array}$ & $\begin{array}{l}27,5 \\
0\end{array}$ & $\begin{array}{l}\text { pun } \\
\text { del }\end{array}$ & & & \\
\hline 8 & sono & 18,6 & son & $\begin{array}{l}\text { cama } \\
\text { almofada }\end{array}$ & $\begin{array}{l}27,7 \\
2,0\end{array}$ & $\begin{array}{l}\text { cam } \\
\text { alm }\end{array}$ & $\begin{array}{l}\text { indispensável } \\
\text { activo }\end{array}$ & $\begin{array}{l}0 \\
2,0\end{array}$ & $\begin{array}{l}\text { ind } \\
\text { act }\end{array}$ & & & \\
\hline
\end{tabular}

Nota: \% C-Percentagem de completamento de inícios de palavras (Linha de base)

as listas iniciavam com uma palavra processada ao nível superficial, todas concluíam também com uma palavra processada ao nível profundo. Cada associado foi exposto durante 2 segundos, sendo seguindo de uma questão. Após a resposta no teclado era apresentado um novo associado. Caso o participante não respondesse em 4 segundos era apresentado o associado seguinte.

Os participantes dos dois grupos com evocação prévia foram ainda informados de que a apresentação das palavras e respectivas questões seria feita por blocos, sendo que no final de cada bloco veriam a palavra "evocação" que indicaria o momento em que registariam no caderno de respostas as palavras apresentadas anteriormente. Além disso, eram advertidos a evocar todas as palavras que tinham visto sem tentar adivinhar. A tarefa de evocação teve uma duração de 90 segundos para cada lista.

As instruções de codificação transmitidas aos dois grupos sem evocação prévia consistiram nas mesmas dos restantes grupos, variando apenas no que concerne à realização da tarefa distractiva. Deste modo, os participantes foram informados de que a apresentação das palavras e respectivas questões seria feita por blocos e que no final de cada um deles surgiriam no ecrã operações de adição ou de subtracção (e.g., "122 + $19=$ ?"), cabendo-lhes efectuar o cálculo de cada operação apresentada e dizer o seu resultado em voz alta para ser registado. Os participantes dispunham de apenas 6 segundos para efectuar cada cálculo. A tarefa distractiva envolvia 15 operações, de modo que tivesse a mesma duração da tarefa de evocação.

Os participantes dos grupos de recuperação explícita foram instruídos de que após a realização da tarefa de evocação e da tarefa distractiva seriam apresentadas 56 palavras, sendo a sua tarefa determinar se cada uma das palavras lhes tinha, ou não, sido previamente apresentada, para esse efeito deveriam utilizar as teclas "S" (de "sim") e "N" (de "não").
Dispunham, para esse efeito, de apenas de 2 segundos por palavra. Após a emissão da resposta era imediatamente apresentado um novo item.

Por seu lado, os participantes dos grupos de recuperação implícita, após a evocação prévia ou a tarefa distractiva, foram instruídos de que seriam apresentados inícios de palavras, devendo dizer em voz alta a primeira palavra que lhes ocorresse à mente e que permitisse completar correctamente o início apresentado. Eram também advertidos a procurar ser rápidos na emissão das respostas pois teriam somente 2 segundos para o fazer. As palavras ditas pelos participantes eram registadas pela experimentadora. Após a emissão da resposta, era exposto o início de palavra seguinte.

\section{Resultados}

Foram consideradas evocações correctas, para além das palavras apresentadas, todas aquelas que correspondiam a alterações aos associados em género (e.g., "preto" em vez de "preta" na Lista 6), número (e.g., "sentimentos" em vez de "sentimento" na Lista 5), tempo verbal (e.g., "despertou" em vez de "desperto" na Lista 8) ou outras, desde que não afectassem substancialmente a morfologia do associado apresentado. Nos restantes casos, as palavras evocadas foram classificadas como intrusões (e.g., "alentejano" em vez de "Alentejo" na Lista 2, "despertador" em vez de "desperto" na Lista 8). Além disso, quando a evocação de associados ou de itens críticos não teve lugar após a apresentação de listas às quais correspondiam, essas respostas foram igualmente consideradas como intrusões.

Atendendo a que os grupos com evocação prévia não se distinguiam foram considerados um único grupo para efeitos de tratamento dos dados nesta tarefa de memória. 


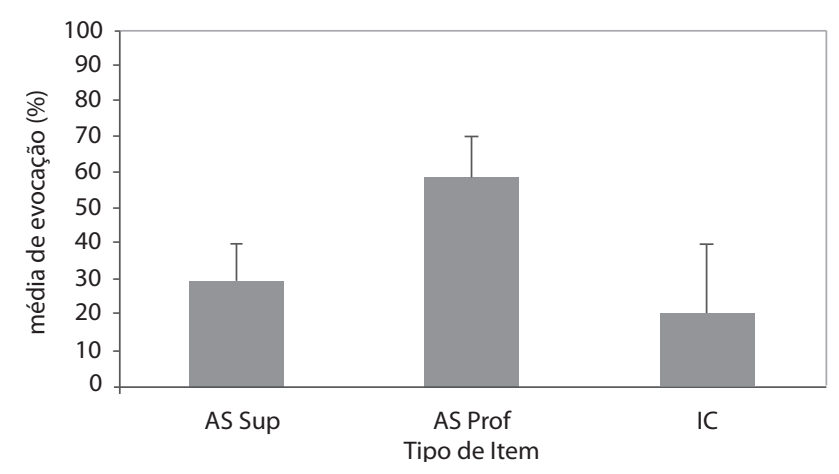

Figura 1. Evocação de associados (AS) em função dos níveis de processamento superficial (Sup) e profundo (Prof) e evocação de itens críticos (IC)

Os resultados relativos à evocação correcta e à evocação de itens críticos encontram-se na Figura 1.

Como é possível observar, quanto ao efeito dos níveis de processamento verificamos uma retenção mais elevada dos associados processados a nível profundo. Com vista a testar o efeito da variável nível de processamento realizámos um teste $\mathrm{t}$ para amostras emparelhadas que revelou diferenças significativas $[t(38)=14,67 ; p=0,001 ; d=2,51]$.

Posteriormente, analisámos a evocação em função do tipo de item $\left(\mathrm{M}_{\text {associados }}=44,3 \%\right.$ e $\left.\mathrm{M}_{\text {itens críticos }}=21,2 \%\right)$ tendo verificado igualmente a existência de diferenças significativas $[t(38)=8,30 ; p=0,001 ; d=1,09]$.

O desempenho dos participantes na tarefa de reconhecimento pode ser observado na Figura 2.

Independentemente da evocação prévia, as palavras codificadas de modo profundo foram, em média, mais reconhecidas do que aquelas que foram objecto de codificação superficial $\left(\mathrm{M}_{\text {profundo }}=79,2 \%\right.$ e $\left.\mathrm{M}_{\text {superficial }}=67,9 \%\right)$. Porém, considerando a variável evocação prévia constamos que no grupo em que esta ocorreu não se observam diferenças apreciáveis em função do nível de processamento $\left(\mathrm{M}_{\text {profundo }}\right.$ $=76,3 \%$ e $\left.\mathrm{M}_{\text {superficial }}=75,7 \%\right)$, contrariamente ao que se verifica no grupo sem evocação prévia $\left(M_{\text {profundo }}=81,9\right.$ e $\left.M_{\text {superficial }}=60,6\right)$.

A realização de uma ANOVA bifactorial mista 2 (nível de processamento: superficial vs. profundo) x 2 (evocação prévia: presente vs. ausente), com medidas repetidas no primeiro factor, permitiu-nos confirmar que o efeito principal nível de processamento e que a interacção entre este e a evocação prévia são estatisticamente significativos $[F(1$, $37)=9,14 ; p=0,005 ; d=0,20$ e $F(1,37)=8,08 ; p=0,007$; $d=0,18$, respectivamente]. Já o factor evocação prévia não se revelou uma variável com efeitos marcantes no reconhecimento de associados.

Para explorarmos a natureza do efeito de interacção, ou seja, a presença do efeito do nível de processamento somente no grupo sem evocação prévia, verificámos, para cada participante, se os êxitos correspondiam a palavras previamente evocadas e qual o grau de profundidade com que tinham sido codificadas. Dessa análise pudemos apurar que, relativamente às palavras evocadas e posteriormente reconhecidas pelos participantes, em média 48,7\% tinham sido processadas a nível profundo, verificando-se uma média idêntica de palavras processadas superficialmente $(M=49,3 \%)$. Podemos concluir que, apesar da codificação profunda ter favorecido a recuperação no primeiro momento de avaliação (i.e., na tarefa de evocação dos grupos com evocação e na tarefa de reconhecimento do grupo sem evocação) esta variável deixou de ser determinante no segundo momento de avaliação (i.e., na tarefa de reconhecimento do grupo com evocação). Assim se explica que a evocação

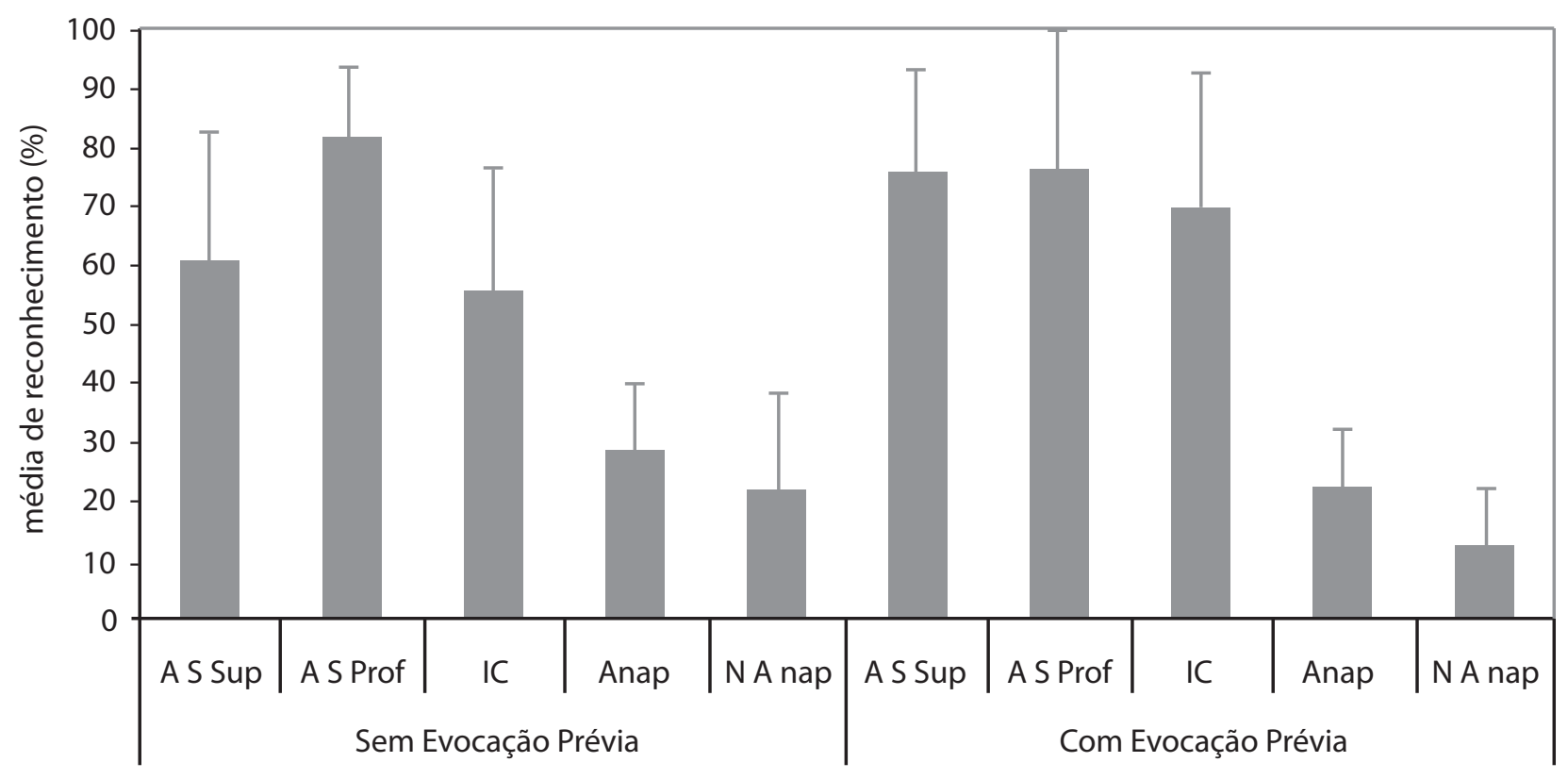

Figura 2. Êxitos (AS) em função dos níveis de processamento superficial (Sup) e profundo (Prof) e da presença e ausência de evocação prévia, falsos alarmes relativos a itens críticos (IC), palavras associadas não apresentadas (Anap) e palavras não associadas não apresentadas (NAnap) em função da ausência e presença de evocação prévia 


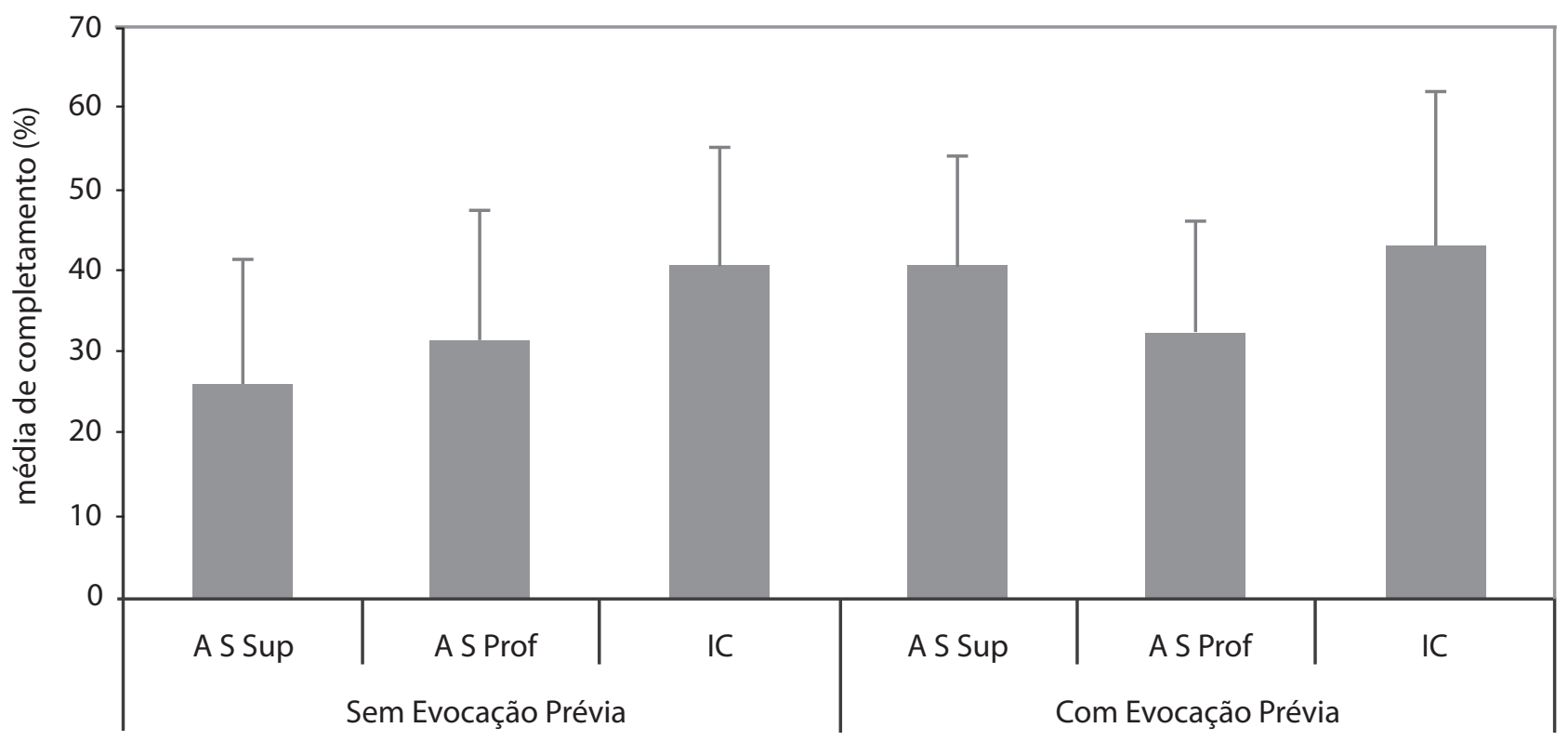

Figura 3. Completamento de inícios de palavras com associados (AS) em função dos níveis de processamento superficial (Sup) e profundo (Prof) e da ausência e presença de evocação prévia; completamento de inícios de palavras com itens críticos (IC) em função da ausência e presença de evocação prévia

prévia tenha resultado na anulação do efeito de nível de processamento neste último grupo.

No que concerne à média de êxitos e de falsos alarmes relativos a itens críticos em função da presença ou ausência de evocação prévia é possível verificar que o grupo que realizou a evocação prévia reconheceu, em média, mais associados $\left(\mathrm{M}_{\text {explícita com evocação }=} 76,0 \%\right.$ e $\left.\mathrm{M}_{\text {explíita sem evocação }}=71,3 \%\right)$ e cometeu mais falsos alarmes relativamente a itens críticos que o de recuperação explícita sem evocação prévia. Por outro lado, o reconhecimento de associados foi maior que o falso alarme relativamente a itens críticos não só em cada grupo, mas também independentemente do grupo $\left(\mathrm{M}_{\text {associados }}=73,6 \%\right.$ e $\mathrm{M}_{\text {itens críticos }}=62,5 \%$ ).

A ANOVA bifactorial mista 2 (tipo de item: associados vs. itens críticos) x 2 (evocação prévia: presente vs. ausente), com medidas repetidas no primeiro factor, demonstrou que somente o factor tipo de item foi estatisticamente significativo $[F(1,37)=11,65 ; p=0,002 ; d=0,24]$, pelo que nem a evocação prévia ou a interacção dos factores contribuíram para diferenciar significativamente os resultados.

Analisando agora a média de falsos alarmes em função do tipo de item e da realização, ou não, da evocação prévia, é possível verificar que a média de falsos alarmes relativos a itens críticos é consideravelmente mais elevada que a dos outros dois tipos de distractores.

Efectuámos uma ANOVA bifactorial mista 3 (tipo de item: itens críticos vs. associados não apresentados vs. não associados não apresentados) x 2 (evocação prévia: presente vs. ausente), com medidas repetidas no primeiro factor. Esta análise mostrou que o factor tipo de item foi significativo $[F(2,74)=123,37 ; p=0,001 ; d=0,77]$, bem como, a interacção entre os factores $[F(2,74)=8,62 ; p=$ $0,002 ; d=0,19]$, mas não o factor evocação após cada lista. Para identificarmos o par ou pares de médias do factor tipo de item que diferiam entre si, realizámos testes post-hoc, tendo encontrado diferenças estatisticamente significativas entre os três pares de médias $(p=0,001)$.

Com base na Figura 3 podemos analisar a média de completamento de inícios de palavras em função do nível de processamento.

Independentemente da realização ou não da tarefa de evocação, as médias de completamento de palavras com associados em cada nível de processamento apenas diferem ligeiramente $\left(\mathrm{M}_{\text {superficial }}=33,2 \%\right.$ e $\left.\mathrm{M}_{\text {profundo }}=32,0 \%\right)$. Quanto ao impacto desta variável em cada grupo verificamos que o completamento de inícios de palavras processadas profundamente foi, em média, sensivelmente o mesmo em ambos os grupos. No que concerne ao nível de processamento superficial constatamos que o grupo que realizou a tarefa de evocação completou mais inícios de palavras com associados.

Independentemente do nível de processamento das palavras, o grupo de recuperação implícita com evocação prévia completou em média mais vezes os inícios de palavras com associados $(\mathrm{M}=36,6 \%)$ que o grupo de recuperação implícita sem evocação prévia $(\mathrm{M}=28,9 \%)$.

A ANOVA bifactorial mista 2 (nível de processamento: superficial vs. profundo) x 2 (evocação prévia: presente vs. ausente), com medidas repetidas no primeiro factor, confirmou a ausência do factor principal nível de processamento e a presença do factor principal evocação prévia $[F(1,39)=$ $4,60 ; p=0,038 ; d=0,11]$ e de interacção $[F(1,39)=5,55$; $p=0,024 ; d=0,13]$.

Embora a variável evocação prévia tenha contribuído para o aumento do número de inícios de palavras completados com associados, a diferença só é expressiva no que se refere aos associados processados superficialmente. Deste modo, analisámos em relação ao grupo de recuperação implícita com evocação prévia o completamento de inícios de palavras com associados que tinham sido previamente codificados em cada nível de processamento. Esta análise permitiu apurar que 
Tabela 3. Linhas de base relativas ao completamento de inícios de palavras com associados e itens críticos (LB), completamento de inícios de palavras com esses itens (ASS e IC), efeitos de primação directa e de primação semântica em função da presença e ausência de evocação prévia; dados em percentagem

\begin{tabular}{ccccccc}
\hline & & Linhade Base & \multicolumn{2}{c}{ Completamento de inícios de palavras } & \multicolumn{2}{c}{ Primação } \\
\cline { 3 - 7 } & & $(\mathrm{LB})$ & $\begin{array}{c}\text { Sem Evocação } \\
\text { Prévia (ASS) }\end{array}$ & $\begin{array}{c}\text { Com Evocação } \\
\text { Prévia (IC) }\end{array}$ & $\begin{array}{c}\text { Sem Evocação } \\
\text { Prévia (ASS - LB) }\end{array}$ & $\begin{array}{c}\text { Com Evocação } \\
\text { Prévia (IC - LB) }\end{array}$ \\
\hline \multirow{2}{*}{ Associados } & $\mathrm{M}$ & 18,0 & 28,9 & 36,6 & 10,9 & 18,6 \\
\hline \multirow{2}{*}{ Itens Críticos } & $\mathrm{DP}$ & 19,11 & 13,03 & 9,6 & 13,03 & 22,2 \\
\cline { 2 - 7 } & $\mathrm{M}$ & 18,3 & 40,5 & 43,1 & 14,74 & 24,8 \\
\hline
\end{tabular}

dentre os inícios de palavras testados, os associados correspondentes reportavam-se em maior número a itens processados superficialmente e posteriormente evocados $\left(\mathrm{M}_{\text {superficial }}\right.$ $=20,6 \% \mathrm{e} \mathrm{M}_{\text {profundo }}=13,1 \%$ ). Por conseguinte, relacionamos a interacção estatística observada com a desproporção entre a quantidade de pistas apresentadas (relativas a associados) em cada nível de processamento, e não ao processamento propriamente dito.

Analisando agora, as médias de completamento de inícios de palavras com associados e itens críticos em cada grupo constata-se que o grupo de recuperação implícita com evocação prévia completou mais inícios de palavras com associados, bem como, itens críticos, sendo por conseguinte, aquele que registou uma média mais elevada de completamento de palavras independentemente do tipo de item $\left(\mathrm{M}_{\text {implicita com }}\right.$ evocacãa $=38,8 \%$ e $\left.\mathrm{M}_{\text {implicita sem evocacão }}=32,7 \%\right)$. No que toca ao tipo de início de palavra verificou-se, em média, um maior completamento de inícios de palavras com itens críticos ( $\mathrm{M}$ $=41,8 \%)$ do que com associados $(\mathrm{M}=32,6 \%)$.

A ANOVA bifactorial mista 2 (tipo de início de palavra: associados vs. itens críticos) x 2 (evocação prévia: presente vs. ausente), com medidas repetidas no primeiro factor, demonstrou que somente o factor tipo de início de palavra foi estatisticamente significativo $[F(1,39)=7,72 ; p=0,008, d$ $=0,17]$, sendo que nem a evocação prévia ou a interacção dos factores concorreram de forma significativa para as diferenças entre os grupos.

Finalmente, o efeito de primação foi calculado com base na diferença entre as médias de completamento de inícios de palavras com associados e com itens críticos e as respectivas linha de base ${ }^{3}$.

Como se pode constatar, as médias de completamento de inícios de palavras com associados e itens críticos foram superiores às respectivas linhas de base (Tabela 3 ) verificando-se, assim, o efeito de primação directa e semântica, respectivamente.

Com vista a apurar se as diferenças entre as percentagens médias de completamento de palavras associadas e as linhas de base para esses mesmos associados eram ou não significativas procedeu-se à realização de testes $t$ para uma amostra. A diferença de médias revelou ser estatisticamente significativa tanto no grupo de recuperação implícita com evocação prévia

3 A linha de base diz respeito à média das frequências de completamento de radicais com as mesmas palavras que constituem as listas da nossa tarefa (cf. Pimentel \& Albuquerque, 2007). $[t(19)=8,67 ; p=0,001 ; d=0,95]$, como no de recuperação implícita sem evocação prévia $[t(20)=3,82 ; p=0,001, d$ $=0,53]$. Encontramos as mesmas diferenças significativas relativamente aos itens críticos no grupo de recuperação implícita com evocação prévia $[t(19)=5,77 ; p=0,001, d=$ $2,78]$, assim como, no grupo de recuperação implícita sem evocação prévia $[t(20)=6,90 ; p=0,001 ; d=2,44]$.

Finalmente, pudemos verificar que a primação de itens críticos $(M=23,5 \%)$ foi estatisticamente superior à de associados $(\mathrm{M}=14,6 \%)$. Tendo em vista testar os efeitos principais e de interacção das variáveis tipo de início de palavra e evocação prévia efectuámos uma ANOVA bifactorial mista 2 (tipo de início de palavra: associados vs. itens críticos) x 2 (evocação prévia: presente vs. ausente), com medidas repetidas no primeiro factor, a qual confirmou que o factor tipo de item foi estatisticamente significativo $[F(1$, 39) $=7,23 ; p=0,010 ; d=0,16]$, enquanto que a evocação prévia ou interacção dos factores não diferenciaram significativamente os grupos.

\section{Discussão}

Na tarefa de evocação constatou-se que os associados codificados profundamente foram significativamente mais evocados do que os codificados a nível superficial; efeito que também se verificou na tarefa de reconhecimento no grupo de recuperação explícita sem evocação prévia.

De acordo com o racional teórico da teoria dos níveis de processamento (Craik \& Lockhart, 1972), o processamento semântico dos itens que produz uma melhoria do grau de retenção deveria, simultaneamente, conduzir não a um aumento (como se verifica) mas a uma diminuição na produção de falsas memórias, dado que este nível de processamento tende a favorecer uma memória robusta e precisa. Uma outra explicação, baseada no processo de propagação da activação, sugere a importância da intensidade com que um conceito é activado na rede semântica para a sua recuperação futura. Quanto mais profundamente os itens associados são processados, tanto maior será a activação entre esses itens, assim como, entre outros itens associados, designadamente o item crítico. A maioria dos estudos que manipula os níveis de processamento no paradigma DRM (e.g., Rhodes \& Anastasi, 2000; Thapar \& McDermott, 2001; Toglia, Neuschatz, \& Goodwin, 1999) tem vindo a apoiar fortemente esta explicação baseada na activação. Efectivamente, quando todos os itens de uma lista de palavras associadas são processados profundamente constata-se um aumento na capacidade de 
recuperação, bem como, um incremento de falsas memórias (em tarefas de evocação e de reconhecimento).

Com base nos processos metacognitivos também poderia supor-se que a codificação profunda conduzisse à rejeição dos itens críticos, em particular a partir do mecanismo de monitorização pelo diagnóstico que consiste na rejeição de uma memória face à incapacidade de recuperar a informação esperada (Gallo, 2006) (e.g., "Eu não vi esta palavra, senão lembrar-me-ia da sua frase"). Porém, estes mecanismos parecem ser mais eficazes quando os participantes se apoiam em evidências ou pistas mais fortes, como por exemplo, o conhecimento relativamente ao efeito DRM (Gallo, Roediger, \& McDermott, 2001; Gallo, Roberts, \& Seamon, 1997; McDermott \& Roediger, 1998); as características perceptivas distintivas e a modalidade sensorial em que os itens foram apresentados (e.g., apresentação de figuras) (Schacter, Israel, \& Racine, 1999; Israel \& Schacter, 1997).

Podemos ainda acrescentar que o tipo de tarefa de codificação interage com o tipo de material a aprender (transferência apropriada de processamento). Com efeito, a correspondência entre as características do material e o tipo de processamento favorece uma maior activação dos itens apresentados, contrariamente ao que ocorre quando o mesmo material, caracterizado pela forte componente semântica, é processado com base numa tarefa não semântica como a contagem de vogais das palavras. Esta perspectiva foi explorada num estudo de Chan, McDermott, Watson e Gallo (2005) em que na primeira experiência apresentaram listas de associados fonológicos e na segunda recorreram também à apresentação de listas de associados semânticos, tendo-se verificado que na tarefa de reconhecimento a sobreposição entre as características do tipo de material e do tipo de tarefa de processamento favoreceu a recuperação para as palavras previamente apresentadas.

Relativamente à manipulação da variável evocação prévia (presente vs. ausente), verificou-se que na tarefa de reconhecimento a evocação prévia conduziu à anulação do efeito do nível de processamento. Após a evocação, a profundidade de processamento deixa de constituir uma variável diferenciadora na probabilidade de recuperação dos itens, eventualmente pelo facto deste processo se apoiar privilegiadamente na memória do episódio de evocação (mais recente) e não na do episódio de codificação. Por outro lado, a evocação contribui para a avaliação de que as palavras foram apresentadas, independentemente da memória que os participantes tenham das mesmas (em função do seu nível de processamento). De igual modo, a evocação de itens críticos terá conduzido à avaliação de que pertenceriam às listas apresentadas, assim como, a uma maior activação destes itens, o que também explicará o aumento deste tipo de falso alarme no grupo com evocação, comparativamente aos outros falsos alarmes.

No que concerne ao efeito da evocação prévia na tarefa de completamento de inícios de palavras verificámos que, pese embora esta variável tenha favorecido significativamente o número de inícios de palavras completados com associados, este efeito só foi expressivo relativamente aos itens codificados a nível superficial. Relacionamos este resultado com o facto dos inícios de palavras testados na tarefa de completamento de memória implícita coincidirem em maior número com os itens processados superficialmen- te e posteriormente evocados. Por conseguinte, teria sido necessário assegurar relativamente aos inícios palavras de associados previamente evocados por cada participante, que metade deles correspondesse a cada nível de processamento. Apesar do impacto da variável evocação prévia, salientamos que a ausência do efeito de nível de processamento afasta a possibilidade do completamento de inícios de palavras ter sido realizado de forma explícita.

O efeito da evocação prévia dilui-se, porém, quando se considera o tipo de item (associados e itens críticos) e não o nível de processamento. Assim, o episódio de evocação não parece ter favorecido a activação daqueles itens na rede semântica, o que pensamos estar associado à evocação consideravelmente baixa de associados e de itens críticos, que resultou provavelmente da manipulação intra-lista do nível de processamento dos associados. A codificação profunda de somente metade dos itens de cada lista terá contribuído não só para uma maior consolidação do traço de memória de apenas parte dos associados apresentados, como também terá contribuído menos intensamente para a activação do item crítico durante a codificação dos associados. Este é, tanto quanto sabemos, o primeiro estudo a manipular deste modo o nível de processamento no paradigma DRM.

Antes de discutirmos os resultados obtidos na tarefa de completamento de radicais de palavras, importa salientar que a melhoria da evocação e reconhecimento dos associados processados a nível profundo no grupo de recuperação explícita sem evocação prévia confirma que a manipulação da variável nível de processamento foi bem sucedida, condição sem a qual não seria possível avaliar se a recuperação na tarefa de completamento de inícios de palavras tinha sido contaminada, ou não, por estratégias explícitas. Por conseguinte, nestas condições a ausência do efeito do nível de processamento na primação dos associados afigura-se um resultado particularmente relevante do nosso estudo. Uma vez que a melhoria da retenção associada ao processamento profundo da informação só se verificou quando a tarefa de memória foi de natureza explícita, podemos concluir que a tarefa de completamento de inícios de palavras foi realizada de forma implícita, isto é, sem recurso a processos de monitorização.

Nesta experiência obtivemos também a primação significativa do item crítico. Assim, conclui-se que a produção de falsas memórias é independente da recordação intencional ou consciente do episódio de codificação das listas de associados. Além disso, este efeito não pode atribuir-se exclusivamente a erros de monitorização da fonte, uma vez que esta não é relevante para a recuperação dos itens em tarefas de memória implícita.

Os nossos dados corroboram a possibilidade da activação não consciente dos itens críticos em tarefas perceptivas de completamento de inícios que foi já demonstrada por outros autores (e.g., Hicks \& Starns, 2005, Experimento 1; McKone \& Murphy, 2000, Experimentos 1 e 3; Smith et al., 2002, Experimentos 2 e 3; Tajika \& Hamajima, 2002; Tajika et al., 2005); efeito de primação que nem sempre é obtido quando são usadas tarefas de memória implícita de decisão lexical e de identificação (e.g., McKone, 2004; Zeelenberg \& Pecher, 2002, Experimentos 1, 2 e 4), as quais parecem privilegiar, mais ainda, o acesso às características físicas dos estímulos. De notar que a melhoria do completamento de itens críticos 
e de associados foi comparada com linhas de base para a qual contribuíram as mesmas palavras testadas na nossa experiência. Mais importante ainda, é a sua constatação face aos efeitos dissociados obtidos com a manipulação do nível de processamento, afastando assim a hipótese da contaminação explícita do teste. Por conseguinte, os resultados da nossa experiência não confirmam que a recuperação implícita dos itens críticos só possa ser obtida em testes de memória perceptivos (como é o caso da tarefa de completamento de inícios de palavras) quando a aprendizagem dos itens das listas é intencional, levando a que os participantes tenham consciência do teste durante a realização da tarefa de memória implícita, como afirmam McBride et al. (2006).

Verificamos na presente experiência que os participantes completaram, em média, significativamente mais inícios de palavras com itens críticos $(\mathrm{M}=41,8)$ do que com associados $(\mathrm{M}=32,6)$, tendência que foi confirmada quando se compararam os efeitos de primação que resultam de cada tipo de item, verificando-se que a primação semântica $(M=23,5)$ foi, não só significativa, como estatisticamente superior à primação directa $(M=14,6)$. Uma vez que a tarefa de evocação não contribuiu para um aumento significativo de completamento de inícios de palavras com itens críticos, não podemos assumir que este resultado decorreu de uma parte significativa destes itens ter sido evocada, pois nessas circunstâncias a activação teria sido, simultaneamente, semântica (devido à sua relação com as palavras apresentadas) e directa (dado os itens críticos terem sido fisicamente produzido na tarefa de evocação). Assim sendo, o valor elevado de primação de itens críticos exprime, efectivamente, a activação semântica do tema das listas, corroborando a possibilidade dos itens críticos serem activados em tarefas perceptivas de completamento de inícios de palavras. Além disso, este resultado ilustra a activação somativa dos itens críticos no momento da codificação das respectivas listas, que resulta da propagação da activação que tem origem em cada associado.

\section{Referências}

Albuquerque, P. B. (2001). Memória implícita e processamento: Do subliminar à formação de imagens. Braga: Centro de Estudos em Educação e Psicologia da Universidade do Minho.

Albuquerque, P. B. (2005). Produção de evocações e reconhecimentos falsos em 100 listas de palavras associadas portuguesas. Laboratório de Psicologia, 3, 3-12.

Albuquerque, P. B., \& Pimentel, E. (2005). Impacto da inibição do efeito de recência na produção de falsas memórias em listas de associados. Psicologia Educação e Cultura, 9(1), 69-87.

Busnello, R. H. D. (2010). Memória implícita, priming e falsas memórias. In L. M. Stein (Org.), Falsas memórias: Fundamentos científicos e suas aplicações clínicas e jurídicas (pp. 101-132). São Paulo: Artmed Editora.

Chan, J. C. K., McDermott, K. B., Watson, J. M., \& Gallo, D. A. (2005). The importance of material-processing interactions in inducing false memories. Memory \& Cognition, 33(3), 389-395.

Craik, F. I. M., \& Lockhart, R. S. (1972). Levels of processing: A framework for memory research. Journal of Verbal Learning and Verbal Behavior, 11, 671-684.
Deese, J. (1959). On the prediction of occurrence of particular verbal intrusions in immediate recall. Journal of Experimental Psychology, 58(1), 17-22.

Gallo, D. A. (2006). Associative illusions of memory: false memory research in DRM and related tasks. New York: Psychology Press.

Gallo, D. A., Roberts, M. J., \& Seamon, J. G. (1997). Remembering words not presented in lists: Can we avoid creating false memories? Psychonomic Bulletin \& Review, 4(2), 271-276.

Gallo, D. A., Roediger, H. L., \& McDermott, K. B. (2001). Associative false recognition occurs without strategic criterion shifts. Psychonomic Bulletin \& Review, 8(3), 579-586.

Hicks, J. L., \& Starns, J. J. (2005). False memories lack perceptual detail: Evidence from implicit word-stem completion and perceptual identification tests. Journal of Memory and Language, 52, 309-321.

Israel, L., \& Schacter, D. L. (1997). Pictorial encoding reduces false recognition of semantic associates. Psychonomic Bulletin \& Review, 4(4), 577-581.

McBride, D. M., Coane, J. H., \& Raulerson III, B. A. (2006). An investigation of false memory in perceptual implicit tasks. Acta Psychologica, 123, 240-260.

McDermott, K. B. (1997). Priming on perceptual implicit memory tests can be achieved through presentation of associates. Psychonomic Bulletin \& Review, 4(4), 582-586.

McDermott, K. B., \& Roediger, H. L. (1998). Attempting to avoid illusory memories: Robust false recognition of associates persists under conditions of explicit warnings and immediate testing. Journal of Memory and Language, 39, 508-520.

McKone, E. (2004). Distinguishing true from false memories via lexical decision as a perceptual implicit test. Australian Journal of Psychology, 56(1), 42-49.

McKone, E., \& Murphy, B. (2000). Implicit false memory: Effects of modality and multiple study presentations on long-lived semantic priming. Journal of Memory and Language, 43, 89-109.

Pimentel, E., \& Albuquerque, P. B. (2007). Normas completamento de radicais de palavras portuguesas. Laboratório de Psicologia, 5(1), 65-80.

Pinho, M. S., Simões, M. R., Beato, M. S., \& Díez, E. (2004). Estudo exploratório sobre a resistência ao efeito de evocação falsa imediata no paradigma DRM. Psicologia e Educação, 3(2), 57-69.

Rhodes, M. G., \& Anastasi, J. S. (2000). The effects of a levels-ofprocessing manipulation on false recall. Psychonomic Bulletin \& Review, 7(1), 158-162.

Roediger, H. L. (1990). Implicit memory: A commentary. Bulletin of the Psychonomic Society, 28(4), 373-380.

Roediger, H. L., \& Blaxton, T. A. (1987). Effects of varying modality, surface features, and retention interval on priming in word fragment completion. Memory \& Cognition, 15(5), 379-388.

Roediger, H. L., \& McDermott, K.B. (1993). Implicit memory in normal human subjects. In F. Boller \& J. Grafman (Eds.), Handbook of neuropsychology, Vol. 8 (pp. 63-131). Amsterdam: Elsevier.

Roediger, H. L., \& McDermott, K. B. (1995). Creating false memories: Remembering words not presented in lists. Journal of Experimental Psychology: Learning, Memory, and Cognition, 21(4), 803-814. 
Roediger, H. L., \& McDermott, K. B. (2000). Distortions of Memory. In E. Tulving \& F. I. M. Craik. (Eds.), The Oxford Handbook of Memory (pp. 149-162). Oxford: Oxford University Press.

Roediger, H. L., Weldon, M. S., \& Challis, B. H. (1989). Explaining dissociations between implicit and explicit measures of retention: A processing account. In H. L. Roediger \& F. I. M. Craik (Eds.), Varieties of memory and consciousness: Essays in honour of Endel Tulving (pp. 3-39). Hillsdale, NJ: Erlbaum.

Schacter, D. L. (1987). Implicit memory: History and current status. Journal of Experimental Psychology: Learning, Memory, and Cognition, 13(3), 501-518.

Schacter, D. L., Dobbins, I. G., \& Schnyer, D. M. (2004). Specificity of priming: A cognitive neuroscience perspective. Nature Reviews Neuroscience, 5, 853-862.

Schacter, D. L., Gallo, D. A., \& Kensinger, E. A. (2007). The cognitive neuroscience of implicit and false memories: Perspectives on processing specificity. In J. S. Nairne (Ed.) The foundations of remembering: Essays in honour of Henry L. Roediger III (pp. 353-378). New York: Psychology Press.

Schacter, D. L., Israel, L., \& Racine, C. (1999). Suppressing false recognition in younger and older adults: The distinctiveness heuristic. Journal of Memory and Language, 40, 1-24.

Smith, S. M., Gerkens, D. R., Pierce, B. H., \& Choi, H. (2002). The roles of associative responses at study and semantically guided recollection at test in false memory: the Kirkpatrick and Deese hypotheses. Journal of Memory and Language, 47, 436-447.

Stein, L. M., Feix, L. F., \& Rohenkohl, G. (2006). Avanços metodológicos no estudo das falsas memórias: Construção e normatização do procedimento de palavras associadas. Psicologia: Reflexão e Crítica, 19(2), 166-176.
Stein, L. M., \& Pergher, G. K. (2001). Criando falsas memórias em adultos por meio de palavras associadas. Psicologia: Reflexão e Crítica, 14(2), 353-366.

Tajika, H., \& Hamajima, H. (2002). Effects of imagery instructions on false memories produced on implicit and explicit memory tests. Japanese Journal of Psychology, 73, 324-331.

Tajika, H., Neumann, E., Hamajima, H., \& Iwahara, A. (2005). Eliciting false memories on implicit and explicit memory tests after incidental learning. Japanese Psychological Research, 47(1), 31-39.

Thapar, A., \& McDermott, K. B. (2001) False recall and false recognition induced by presentation of associated words: Effects of retention interval and level of processing. Memory and Cognition, 29(3), 424-432.

Toglia, M. P., Neuschatz, J. S., \& Goodwin, K. A. (1999). Recall accuracy and illusory memories: When more is less. Memory, 7(2), 233-256.

Zeelenberg, R., \& Pecher, D. (2002). False memories and lexical decision: even twelve primes do not cause long-term semantic priming. Acta Psychologica, 109, 269-284.

Recebido em 02.03.2010

Primeira decisão editorial em 12.05.2010

Versão final em 07.06.2010

Aceito em 11.06.2010 